\title{
Quantum kinetic energy densities: An operational approach
}

\author{
J. G. Muga, ${ }^{1}$ D. Seidel,${ }^{2}$ and G. C. Hegerfeldt ${ }^{2}$ \\ 1 * Departamento de Química-Física, \\ UPV-EHU, Apdo. 644, Bilbao, Spain \\ ${ }^{2}$ Institut für Theoretische Physik, Universität Göttingen, \\ Friedrich-Hund-Platz 1, 3707r Göttingen, Germany
}

\begin{abstract}
We propose and investigate a procedure to measure, at least in principle, a positive quantum version of the local kinetic energy density. This procedure is based, under certain idealized limits, on the detection rate of photons emitted by moving atoms which are excited by a localized laser beam. The same type of experiment, but in different limits, can also provide other non positivedefinite versions of the kinetic energy density. A connection with quantum arrival time distributions is discussed.
\end{abstract}

PACS numbers: 03.65.Nk, 03.65.Xp 


\section{INTRODUCTION}

To obtain an expression for the local density of a quantum observable not diagonal in coordinate representation, one may look for guidance to the corresponding classical case. For a classical dynamical variable, $A(q, p)$, in position-momentum phase space its local density, $\alpha_{A}(x)$, is simply obtained by

$$
\begin{aligned}
\alpha_{A}(x) & =\int d p \rho(x, p) A(x, p) \\
& =\int d q d p \rho(q, p) \delta(x-q) A(q, p)
\end{aligned}
$$

where $\rho(q, p)$ is the phase space density. To quantize this expression one can use

$$
\delta(x-\widehat{x})=|x\rangle\langle x|
$$

and consider, for a point $x$, the operator $\widehat{A}(x)=\widehat{A}|x\rangle\langle x|$, or rather one of its many symmetrizations, as a quantum density for the observable $\widehat{A}$. For a given state $|\psi\rangle$, the expectation value $\langle\psi|\widehat{A}(x)| \psi\rangle$ would then be a candidate for the value of the local density at the

point $x$ of the observable $\widehat{A}$. If $\widehat{A}$ is not diagonal in coordinate representation so that it does not commute with $|x\rangle\langle x|$, there are infinitely many "combinations" (orderings) to construct a quantum density, ${ }^{1.2}$ for example,

$$
\begin{array}{r}
\widehat{A}^{1 / 2}|x\rangle\langle x| \widehat{A}^{1 / 2} \\
\frac{1}{2}(\widehat{A}|x\rangle\langle x|+| x\rangle\langle x| \widehat{A}) \\
\frac{1}{2}\left(\widehat{A}^{1 / 2}|x\rangle\langle x| \widehat{A}^{1 / 2}\right)+\frac{1}{4}(\widehat{A}|x\rangle\langle x|+| x\rangle\langle x| \widehat{A})
\end{array}
$$

The noncommutativity of two observables does not mean that there is only one "true" symmetrization of their product. Different symmetrizations may have a perfectly respectful status as physically observable and measurable quantities, and different orderings may be associated with latent properties that may be realized via different experimental measurement procedures. They may also be related more indirectly to observables and yet carry valuable physical information. An example of this non-uniqueness due to different symmetrizations is the arrival time of a quantum particle at a particular position. Classically the distribution of arrival times would be the flux for particles moving in one direction, but quantum mechanically different quantizations have been proposed. 3.4 
Another important example of this quantum non-uniqueness for a single classical quantity is the kinetic energy density, ${ }^{5.6 .7}$ in which case $\widehat{A}$ becomes the kinetic energy operator, $\widehat{A}=\widehat{p}^{2} / 2 m$. There is no unique definition of a quantum "kinetic energy density", in spite of the relevance of the concept in several fields. The Thomas-Fermi theory provides an early example of a possible realization and application. In density functional theory, it enters as one of the terms of the energy functional to determine the electronic structure of atoms, molecules, solids or fermionic gases, see e.g. Refs. 8,9. In this context it has been used in particular to define a local temperature and identify molecular sites most reactive to electrophilic reagents. ${ }^{8}$ The kinetic energy density also plays a key role in partitioning molecular systems into fragments with well defined energies and virial relations,,$\frac{5.10 .11}{5}$ or to define "intrinsic shapes" of reactants, transition states and products along the course of a chemical reaction. $\frac{12}{2}$ It is moreover a basic quantity in quantum hydrodynamic equations..$^{2,13}$

In all these applications, different quantum versions of the kinetic energy density have been used. The most commonly found cases are the three quantizations considered above, or suitable generalizations thereof. They satisfy different properties and there have been intensive discussions which one is best, but clearly they all are useful in different ways and for different purposes. However, not so much emphasis has been placed on possible procedures of how to measure them.

Many of these arguments and controversies already can be seen in the simple case of the kinetic energy density of a free particle in one dimension. Real, Muga and Brouard ${ }^{14}$ studied and compared three versions of an operator, $\widehat{\tau}(x)$, for the kinetic energy density at a point $x$, associated with different quantizations, namely

$$
\begin{aligned}
\widehat{\tau}^{(1)}(x) & =\frac{\widehat{p} \delta(x-\widehat{x}) \widehat{p}}{2 m}, \\
\widehat{\tau}^{(2)}(x) & =\frac{1}{2}\left[\frac{\widehat{p}^{2}}{2 m} \delta(x-\widehat{x})+\delta(x-\widehat{x}) \frac{\widehat{p}^{2}}{2 m}\right], \\
\widehat{\tau}^{(3)}(x) & =\frac{1}{2}\left[\widehat{\tau}^{(1)}(x)+\widehat{\tau}^{(2)}(x)\right] .
\end{aligned}
$$

The second operator follows from the quantization rule of Rivier. ${ }^{15}$ The corresponding density $\left\langle\widehat{\tau}^{(2)}\right\rangle_{t}$ is given by its, generally time dependent, expectation value and may in principle be obtained operationally by a weak measurement of the kinetic energy post-selected at position $x .^{16.17 .18}$ The third one, which is the average of $\left\langle\widehat{\tau}^{(1)}\right\rangle_{t}$ and $\left\langle\widehat{\tau}^{(2)}\right\rangle_{t}$, corresponds to Weyl's quantization rule. An indirect way to measure $\left\langle\widehat{\tau}^{(3)}\right\rangle_{t}$ for free motion was described 
by Johansen, $\stackrel{19}{=}$ who noticed that the second time derivative of the expectation value of $|\widehat{x}-x|$ is proportional to $\left\langle\widehat{\tau}^{(3)}\right\rangle_{t}$.

In this paper we will provide an operational interpretation of the first expression which, incidentally, is the only positive one among the three, and in fact among a much broader family of quantizations. $\underline{\underline{8}}$ We will use for this end a simple model, originally devised to study time of arrival measurements. ${ }^{20}$ The basic physical idea is to send atoms in their ground state through a region illuminated by a perpendicular laser beam and to measure the resulting fluorescence rate of photons.

\section{KINETIC ENERGY DENSITY, FLUORESCENCE AND ATOMIC ABSORP- TION RATE IN AN IMAGINARY POTENTIAL BARRIER}

The description of photon fluorescence from moving atoms, which are excited by a localized laser beam, is based on the quantum jump approach ${ }^{21.22 .23}$ and has been discussed in detail elsewhere $\underline{\underline{20,24.25}}$ We will only summarize the results which are relevant for the present investigation and assume a "lambda" configuration of three atomic levels in which the laser couples levels 1 and 2 with Rabi frequency $\Omega$, whereas level 2 decays with inverse life time $\gamma$ predominantly and irreversibly to a ground (sink) state $3 \stackrel{26}{\underline{26}}$ For a laser on resonance with the atomic transition, atoms for which no photon is detected ("undetected atoms") are governed by the following effective Hamiltonian

$$
\widehat{H}=\widehat{p}^{2} / 2 m+\frac{\hbar}{2} \Omega(\widehat{x})\{|2\rangle\langle 1|+| 1\rangle\langle 2|\}-\frac{i}{2} \hbar \gamma|2\rangle\langle 2|,
$$

where $\Omega(x)$ is the position dependent Rabi frequency. The evolution of the wave function for undetected atoms simplifies to a one channel Schrödinger equation if $\hbar \gamma$ is large compared to the kinetic energy, with a complex imaginary potential27

$$
U(\widehat{x})=-i \hbar \frac{\Omega(\widehat{x})^{2}}{2 \gamma} .
$$

In this "low saturation regime" the undetected atoms, whose number is proportional to the norm-squared of the wave-function, are in the ground state most of the time, and

the temporal probability density for a photon detection ("detection rate") is given by the decrease of the number of undetected atoms,

$$
\Pi(t)=-d\langle\psi(t) \mid \psi(t)\rangle / d t=-\frac{2}{\hbar}\langle\psi(t)|\operatorname{Im}(U)| \psi(t)\rangle .
$$


This is the basic operational quantity obtained in the modeled experiment. We will relate it, or its normalized version

$$
\Pi_{N}(t)=\frac{\Pi(t)}{\int d t \Pi(t)}
$$

to ideal quantities for the freely moving atom, unperturbed by the laser, by taking certain limits.

First, we consider a square laser beam profile. Then the imaginary potential becomes a barrier of height

$$
V=\hbar \Omega^{2} / 2 \gamma
$$

located between $x=0$ and $x=L$, and the effective Hamiltonian for undetected atoms reads

$$
\widehat{H}=\frac{\widehat{p}^{2}}{2 m}-i V \chi_{[0, L]}(\widehat{x}),
$$

where $\chi_{[0, L]}$ is 1 inside the laser illuminated region and zero outside. The absorption, or detection, rate is found from Eq. (11) and is given by

$$
\Pi(t)=\frac{2 V}{\hbar} \int_{0}^{L} d x|\psi(x, t)|^{2}
$$

To obtain the time development of a wave packet coming in from the left, we solve first the stationary equation

$$
\widehat{H} \phi_{k}=E_{k} \phi_{k}
$$

Using standard matching conditions, the energy eigenfunctions $\phi_{k}$ in the barrier region $0 \leq x \leq L$ for a plane wave coming in from the left with momentum $\hbar k$ are given by

$$
\phi_{k}(x)=\frac{1}{\sqrt{2 \pi}}\left(A_{+}(k) e^{i q x}+A_{-}(k) e^{-i q x}\right)
$$

with $q^{2}=k^{2}+2 i m V / \hbar^{2}$ and

$$
A_{ \pm}(k)=\frac{k(q \pm k) e^{\mp i q L}}{2 k q \cos (q L)-i\left(k^{2}+q^{2}\right) \sin (q L)} .
$$

Writing the initial state as a superposition of eigenfunctions with positive momenta, we obtain

$$
\psi(x, t)=\int_{0}^{\infty} d k \widetilde{\psi}(k) e^{-i \hbar k^{2} t / 2 m} \phi_{k}(x) .
$$

Inserting this into Eq. (15) yields the absorption rate. It is also useful to define the auxiliary freely moving wave packet

$$
\psi_{f}(x, t)=\frac{1}{\sqrt{2 \pi}} \int_{0}^{\infty} d k \widetilde{\psi}(k) e^{-i \hbar k^{2} t / 2 m} e^{i k x}
$$


We will now relate the absorption rate to an ideal distribution by going to the limit $V \rightarrow \infty$. When $V$ is increased, more and more atoms are reflected without being detected, but normalizing the result a finite distribution is obtained in the limit, even though the absorption probability vanishes eventually due to total reflection. For large $V, V \gg \hbar^{2} k^{2} / 2 m$, one has in leading order

$$
\begin{aligned}
q & \simeq \sqrt{2 i m V / \hbar^{2}} \\
A_{+} & \simeq \frac{2 k}{q} \\
A_{-} & \simeq \frac{2 k}{q} e^{2 i q L}, \\
\phi_{k}(x) & \simeq \frac{1}{\sqrt{2 \pi}} \frac{2 k}{q}\left(e^{i q x}+e^{i q(2 L-x)}\right), \quad 0 \leq x \leq L .
\end{aligned}
$$

Integrating over $x$ and neglecting the terms which vanish exponentially, the absorption rate becomes

$$
\Pi(t) \simeq \frac{\hbar^{2}}{\pi m \sqrt{m V}} \int_{0}^{\infty} d k \int_{0}^{\infty} d k^{\prime} \widetilde{\psi}^{*}(k) \widetilde{\psi}\left(k^{\prime}\right) e^{i \hbar\left(k^{2}-k^{\prime 2}\right) t / 2 m} k k^{\prime}
$$

This expression is independent of the barrier length $L$ as a result of the large $V$ limit, so the same result is obtained with an imaginary step potential $-i V \Theta(\widehat{x})$ or with a very narrow barrier.

The normalization constant is given by $\int \Pi(t) d t \simeq 2 \hbar k_{0}(m V)^{-1 / 2}$, where $k_{0}=$ $\int|\widetilde{\psi}(k)|^{2} k d k$, and the normalized absorption rate is

$$
\Pi_{N}(t) \simeq \frac{\hbar}{2 \pi m k_{0}} \int_{0}^{\infty} d k \int_{0}^{\infty} d k^{\prime} \widetilde{\psi}^{*}(k) \widetilde{\psi}\left(k^{\prime}\right) e^{i \hbar\left(k^{2}-k^{\prime 2}\right) t / 2 m} k k^{\prime} .
$$

With the freely moving wave packet $\psi_{f}(x, t)$ this can finally be rewritten in the form

$$
\Pi_{N}(t) \simeq \frac{\hbar}{m k_{0}}\left\langle\psi_{f}(t)|\widehat{k} \delta(\widehat{x}) \widehat{k}| \psi_{f}(t)\right\rangle
$$

Now, the right hand side is just the expectation value at time $t$ of the kinetic energy density $\widehat{\tau}^{(1)}$ evaluated at the origin! Thus, with $p_{0}=k_{0} \hbar$ the initial average momentum we have obtained, in the limit $V \rightarrow \infty$,

$$
\lim _{V \rightarrow \infty} \Pi_{N}(t)=\frac{2}{p_{0}}\left\langle\widehat{\tau}^{(1)}(x=0)\right\rangle_{t} .
$$

Note that the averages are computed with the freely moving wave function and that one obtains the kinetic energy density at an arbitrary point $a$ by shifting the laser region, i.e. by replacing $[0, L]$ by $[a, L+a]$ in Eq. (14) . 
Remark: Instead of normalizing the absorption rate of Eq. (15) by dividing by a constant one can normalize it on the level of operators, 28 which preserves it as a bilinear form. However, in this case the result depends on the constant to which one normalizes. It has been shown in Ref. 25 that if the constant is chosen as 1 then operator normalization of Eq. (15) leads for $V \rightarrow \infty$ to the arrival time distribution of Kijowski. ${ }^{29}$ Now, since the time integral of $\left\langle\widehat{\tau}^{(1)}(x=0)\right\rangle_{t}$ equals $p_{0} / 2=\hbar k_{0} / 2$, it is suggestive to choose $p_{0} / 2$ as normalization constant. Following the approach of Ref. 30 operator normalization of $\Pi(t)$ then leads to $\left\langle\widehat{\tau}^{(1)}(x=0)\right\rangle_{t}$ in the limit $V \rightarrow \infty$.

\section{KINETIC ENERGY DENSITY FROM FIRST PHOTON MEASUREMENT AND DECONVOLUTION}

In the preceding section we had $V=\Omega^{2} / 2 \gamma$, with $\hbar \gamma$ much larger than the kinetic energy. Therefore, the limit $V \rightarrow \infty$ implies a simultaneous change of $\Omega$ and $\gamma$. Experimentally, the Rabi frequency $\Omega$ is easy to adjust, but not the decay rate $\gamma$. To overcome this problem we therefore describe a procedure that allows to keep the value of $\gamma$ fixed.

We again consider the moving-atom laser model but now for the limit $\Omega \rightarrow \infty$ and $\gamma=$ const. In that case, the simplified description of the evolution of the wave function by means of the imaginary potential $U(x)$ is not feasible, and one has to solve the full twochannel problem for the three-level atom with the Hamiltonian in Eq. (92). This has been done in Refs. 20.25, and normalizing with a constant the resulting photon detection rate $\Pi_{N}(t)$ becomes

$$
\Pi_{N}(t) \simeq \frac{\hbar}{2 \pi m k_{0}} \int_{0}^{\infty} d k d k^{\prime} \widetilde{\psi}^{*}(k) \widetilde{\psi}\left(k^{\prime}\right) e^{i \hbar\left(k^{2}-k^{\prime 2}\right) t / 2 m} \frac{\gamma k k^{\prime}}{\gamma+i \hbar\left(k^{2}-k^{\prime 2}\right) / m} .
$$

For $\hbar \gamma$ large compared to the kinetic energy of the incident atom, Eq. (23) is recovered,

but for finite $\gamma$ there is a delay in the detection rate. This can be eliminated by means of a deconvolution with the first-photon distribution $W(t)$ for an atom at rest. ${ }^{20,25}$ The ansatz

$$
\Pi(t)=\Pi_{i d}(t) * W(t)
$$

yields in terms of Fourier transforms

$$
\widetilde{\Pi}_{i d}(\nu)=\frac{\widetilde{\Pi}(\nu)}{\widetilde{W}(\nu)}
$$


with 20,31

$$
\begin{aligned}
\frac{1}{\widetilde{W}(\nu)} & =1+\left(\frac{\gamma}{\Omega^{2}}+\frac{2}{\gamma}\right) i \nu+\frac{3}{\Omega^{2}}(i \nu)^{2}+\frac{2}{\gamma \Omega^{2}}(i \nu)^{3} \\
& \simeq 1+\frac{2 i \nu}{\gamma}, \quad \Omega \rightarrow \infty
\end{aligned}
$$

Inserting this and the Fourier transform of Eq. (26) into Eq. (28), the resulting ideal distribution, after performing the inverse Fourier transform, reads

$$
\Pi_{i d}(t) \simeq \frac{\hbar}{2 \pi m k_{0}} \int_{0}^{\infty} d k \int_{0}^{\infty} d k^{\prime} \widetilde{\psi}^{*}(k) \widetilde{\psi}\left(k^{\prime}\right) e^{i \hbar\left(k^{2}-k^{2}\right) t / 2 m} k k^{\prime}
$$

which is the same expression as the absorption rate of Eq. (23), obtained here operationally for fixed $\gamma$. Naturally,

$$
\Pi_{i d}(t) \simeq \frac{2}{p_{0}}\left\langle\widehat{\tau}^{(1)}(x=0)\right\rangle_{t}
$$

holds as before.

\section{CONNECTION WITH QUANTUM ARRIVAL TIME DISTRIBUTIONS}

Here we briefly discuss a formal connection between the kinetic energy density $\widehat{\tau}^{(1)}(x)$ given in Eq. (24) and Eq. (25) and the arrival-time distribution of Kijowski29,

$$
\Pi_{K}(t)=\frac{\hbar}{m}\left\langle\psi_{f}(t)\left|\widehat{k}^{1 / 2} \delta(\widehat{x}) \widehat{k}^{1 / 2}\right| \psi_{f}(t)\right\rangle
$$

at $x=0$. For wave packets peaked around some $k_{0}$ in momentum space, the operator $\widehat{k}^{1 / 2}$ acting on $\psi_{f}$ in Eq. (32) can be expanded in terms of $\left(\widehat{k}-k_{0}\right)$,

$$
\widehat{k}^{1 / 2}=k_{0}^{1 / 2}+\frac{1}{2} k_{0}^{-1 / 2}\left(\widehat{k}-k_{0}\right)-\frac{1}{8} k_{0}^{-3 / 2}\left(\widehat{k}-k_{0}\right)^{2}+\mathcal{O}\left(\left(\widehat{k}-k_{0}\right)^{3}\right) .
$$

In the following we take $k_{0}$ to be the first moment of the momentum distribution, $k_{0}=$ $\int|\widetilde{\psi}(k)|^{2} k d k$. Inserting the expansion in Eq. (333) into Eq. (32) yields in zeroth order a very simple result,

$$
\Pi_{K}(t)=v_{0}\left|\psi_{f}(0, t)\right|^{2}+\mathcal{O}\left(\widehat{k}-k_{0}\right)
$$

i.e. the particle density times the average velocity $v_{0}=k_{0} \hbar / m$. To first order in $\left(\widehat{k}-k_{0}\right)$ one obtains the flux at $x=0$,

$$
\Pi_{K}(t)=J(0, t)+\mathcal{O}\left(\left(\widehat{k}-k_{0}\right)^{2}\right)
$$


where

$$
J(0, t)=\frac{\hbar}{2 m}\left\langle\psi_{f}(t)|(\widehat{k} \delta(\widehat{x})+\delta(\widehat{x}) \widehat{k})| \psi_{f}(t)\right\rangle,
$$

and to second order the expression

$$
\Pi_{K}(t)=J(0, t)+\frac{1}{2 p_{0}} \Delta(0, t)+\mathcal{O}\left(\left(\widehat{k}-k_{0}\right)^{3}\right)
$$

where

$$
\Delta(0, t)=\left\langle\widehat{\tau}^{(1)}(x=0)\right\rangle_{t}-\left\langle\widehat{\tau}^{(2)}(x=0)\right\rangle_{t}
$$

For states with positive momentum, which we are considering here, the first order, namely the flux, is correctly normalized to one, and so is the second order since the time integral over $\Delta$ is easily shown to vanish. This difference only provides a local-in-time correction to $J$ that averages out globally. Its quantum nature can be further appreciated by the more explicit expression

$$
\frac{1}{2 p_{0}} \Delta=\frac{\hbar^{2}}{8 m p_{0}} \frac{\partial^{2}\left|\psi_{f}(0, t)\right|^{2}}{\partial x^{2}}
$$

which shows the inverse dependence on mass and momentum and the explicit quadratic dependence on $\hbar$.

\section{DISCUSSION}

In Fig. 1, operational and ideal kinetic energy densities are compared for a coherent superposition of two Gaussian wave packets with different mean momenta. They are prepared in such a way that their centers of mass arrive simultaneously at the origin. This enhances the interference among different momentum components and the differences between the distributions. As seen in the figure, the differences between various versions of the quantum kinetic energy density may be quite significant. While $\left\langle\widehat{\tau}^{(1)}(x)\right\rangle_{t}$ is always positive, $\left\langle\widehat{\tau}^{(2)}(x)\right\rangle_{t}$ can become negative in classically forbidden regions for stationary eigenstates of the Hamiltonian, a fact that has been used by Tachibana ${ }^{12}$ to define molecular and reaction shapes. It is perhaps less obvious that this quantity can also be negative as a result of free motion dynamics, as seen in the figure.

The main contribution of this paper has been to point out that, under certain idealizations and limiting conditions, fluorescence experiments can lead to an operational approach to kinetic energy densities. A positive kinetic energy density can be obtained from the "measured" signal in a strong laser limit. With $\Delta$ the difference of the positive density $\left\langle\widehat{\tau}^{(1)}\right\rangle_{t}$ 
and the density $\left\langle\widehat{\tau}^{2}\right\rangle_{t}$ of Rivier, an interesting relation was found for $\Delta$ with the ideal timeof-arrival distribution of Kijowski and with the flux. The latter two can also be obtained operationally under appropriate limits. In a recent review by Ayers, Parr and Nagy ${ }^{\underline{8}}$ on the kinetic energy density, one of the suggestions for future research was the need to study and understand this quantity $\Delta$ better. In a completely different context from the present work, and suitably generalized to three dimensions, $\Delta$ plays a major role in Bader's theory to separate a molecular system into meaningful fragments. ${ }^{5.10 .11}$ Note that, if $\Delta=0,\left\langle\widehat{\tau}^{(3)}\right\rangle_{t}$ becomes also equal to the other two densities considered. Therefore this condition implies a certain "classicality" or coalescence of the multiple quantum possibilities. If $\Delta$ or its integral over some volume are zero, a fragment can be defined with a well defined kinetic energy and virial relations. It is quite striking that, in the second order expansion of the arrival time distribution of Kijowski, $\Delta=0$ implies that $\Pi_{K}$ becomes the flux, which is, as we know, the quantity that plays the role of a arrival time distribution in classical mechanics.

In summary, we think that these results clarify the status, as physically meaningful physical quantities, of several versions of the local kinetic energy densities, and may stimulate experimental research on quantum kinetic energy densities and as well as on arrival times.

\section{Acknowledgments}

This work has been supported by Ministerio de Ciencia y Tecnología, FEDER (BFM20000816-C03-03, BFM2003-01003), UPV-EHU (00039.310-13507/2001), and Acción integrada.

1 L. Cohen and P. Loughlin, J. Mod. Opt. 49, 539 (2002).

2 J. G. Muga, J. P. Palao and R. Sala, Phys. Lett. A 238, 90 (1998).

3 J. G. Muga and C. R. Leavens, Phys. Rep. 338, 353 (2000).

4 J. G. Muga, R. Sala and I. L. Egusquiza (eds) Time in Quantum Mechanics (Springer, Berlin, 2002).

5 L. Cohen, J. Chem. Phys. 70, 788 (1979).

6 L. Cohen, J. Chem. Phys. 80, 4277 (1984).

7 R. W. Robinett, Am. J. Phys. 63, 823 (1995).

8 P. W. Ayers, R. G. Parr and A. Nagy, Int. J. Quant. Chem. 90, 309 (2002). 
9 M. Brack and B. P. van Zyl, Phys. Rev. Lett. 86, 1574 (2001).

10 R. F. W. Bader and P. M. Beddall, J. Chem. Phys. 56, 3320 (1972).

11 R. F. W. Bader, Atoms in Molecules: A Quantum Theory (Clarendon, Oxford, 1990).

12 A. Tachibana, J. Chem. Phys. 115, 3497 (2001).

13 M. W. Thomas and R. F. Snider, J. Stat. Physics 2, 61 (1970).

14 C. C. Real, J. G. Muga and S. Brouard, Am. J. Phys. 65, 157 (1997).

15 D. C. Rivier, Phys. Rev. 83, 862 (1957).

16 Y. Aharonov and L. Vaidman, Phys. Rev. A 41, 11 (1990).

17 Y. Aharonov, S. Popescu, D. Rohrlich, and L. Vaidman, Phys. Rev. A 48, 4084 (1993).

18 L. M. Johansen, Phys. Rev. Lett. 93, 120402 (2004).

19 L. M. Johansen, quant-ph/9804002.

20 J. A. Damborenea, I. L. Egusquiza, G. C. Hegerfeldt and J. G. Muga, Phys. Rev. A 66, 052104 (2002).

21 G. C. Hegerfeldt and T. S. Wilser in Classical and Quantum Systems. Proceedings of the Second International Wigner Symposium 1991, edited by H. D. Doebner, W. Scherer, and F. Schroeck, (World Scientific, Singapore, 1992), p. 104; G. C. Hegerfeldt, Phys. Rev. A 47, 449 (1993).

22 J. Dalibard, Y. Castin and K. Mølmer, Phys. Rev. Lett. 68, 580 (1992).

23 H. Carmichael, An Open Systems Approach to Quantum Optics (Springer, Berlin, 1993).

24 J. A. Damborenea, I. L. Egusquiza, G. C. Hegerfeldt and J. G. Muga, J. Phys. B: At. Mol. Opt. Phys. 36, 2657 (2003).

25 G. C. Hegerfeldt, D. Seidel and J. G. Muga, Phys. Rev. A 68, 022111 (2003).

26 M. K. Oberthaler, R. Abfalterer, S. Bernet, J. Schmiedmayer and A. Zeilinger Phys. Rev. Lett. 77, 4980 (1996).

27 A. Ruschhaupt, J. A. Damborenea, B. Navarro, J. G. Muga, and G. C. Hegerfeldt, Europhys. Lett. 67, 1 (2004).

28 R. Brunetti and K. Fredenhagen, Phys. Rev. A 66, 044101 (2002).

29 J. Kijowski, Rep. Math. Phys. 6, 361 (1974).

30 G. C. Hegerfeldt, D. Seidel, J. G. Muga and B. Navarro, Phys. Rev. A 70, 012110 (2004).

31 M. S. Kim, P. L. Knight, and K. Wodkiewicz, Opt. Comm. 62, 385 (1987). 


\section{FIGURE CAPTION (Fig. 1)}

Comparison of kinetic energy densities at $x=0:\left\langle\widehat{\tau}^{(1)}\right\rangle$ (solid), $\left\langle\widehat{\tau}^{(2)}\right\rangle$ (dashed), $\left\langle\widehat{\tau}^{(3)}\right\rangle$ (dotted) and the operational quantity $p_{0} \Pi_{N}(t) / 2$, see Eqs. (12) and (25), for $V=1.9 \hbar \mu \mathrm{s}^{-1}$, $L=0.21 \mu \mathrm{m}$ (triangles) and $V=950.0 \hbar \mu \mathrm{s}^{-1}, L=0.42 \mu \mathrm{m}$ (circles). The initial wave packet is a coherent combination $\psi=2^{-1 / 2}\left(\psi_{1}+\psi_{2}\right)$ of two Gaussian states for the center-of-mass motion of a single caesium atom that become separately minimal uncertainty packets (with $\Delta x_{1}=\Delta x_{2}=0.031 \mu \mathrm{m}$, and average velocities $\langle v\rangle_{1}=18.96 \mathrm{~cm} / \mathrm{s},\langle v\rangle_{2}=5.34 \mathrm{~cm} / \mathrm{s}$ at $x=0$ and $t=2 \mu \mathrm{s})$. The mass is $2.2 \times 10^{25} \mathrm{~kg}$ and $p_{0}=2.67 \times 10^{-26} \mathrm{~kg} \mathrm{~m} / \mathrm{s}$. 


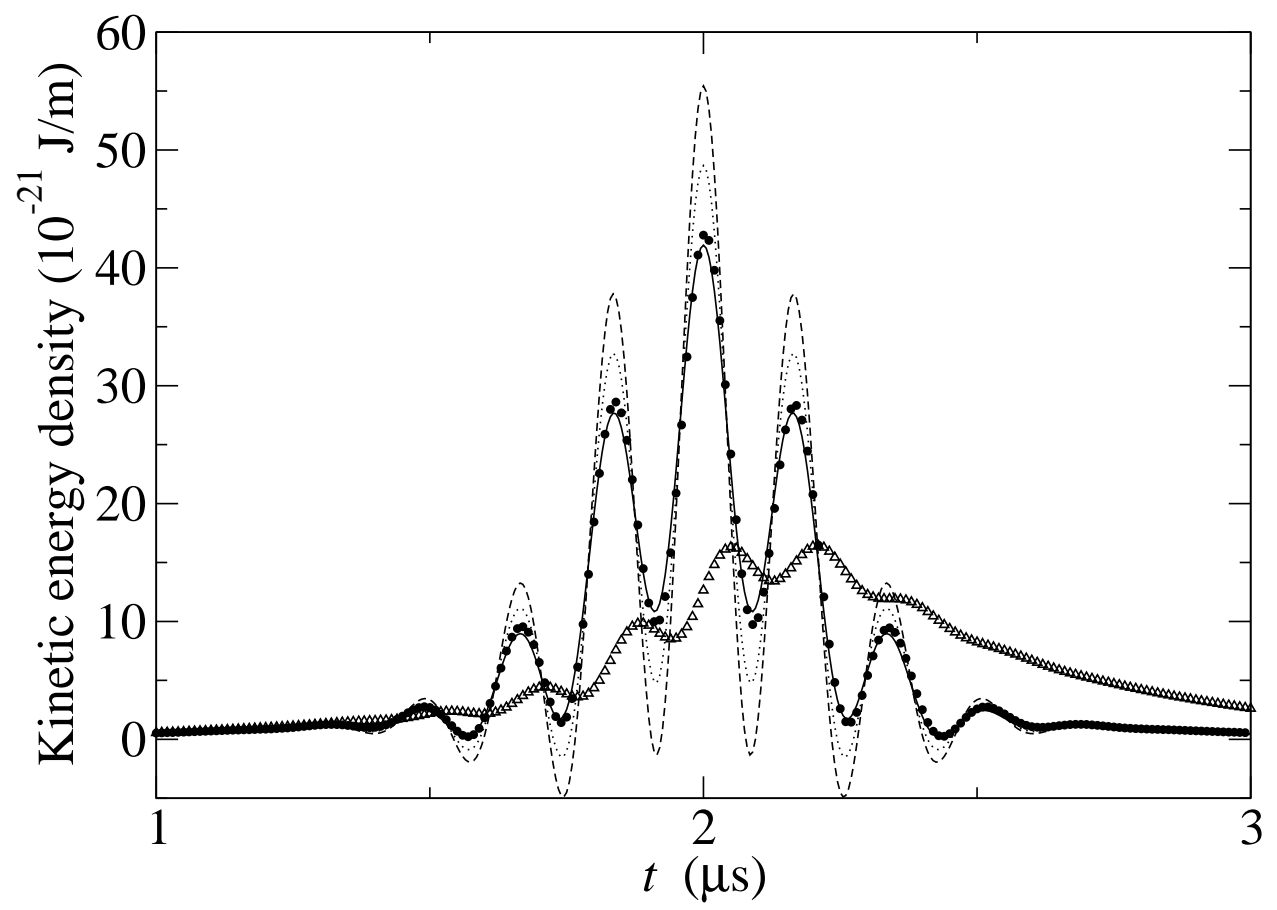

Muga, Seidel and Hegerfeldt, Figure 1 